\title{
Mathematical Structure of Anomalous Dimensions and QCD Wilson Coefficients in Higher Order
}

\author{
J. Blümlein ${ }^{\mathrm{a}}$ \\ a Deutsches Elektronen Synchrotron, DESY, Platanenallee 6, D-15738 Zeuthen, Germany
}

The alternating and non-alternating harmonic sums and other algebraic objects of the same equivalence class are connected by algebraic relations which are induced by the product of these quantities and which depend on their index class rather than on their value. We show how to find a basis of the associated algebra. The length of the basis $l$ is found to be $\leq 1 / d$, where $d$ is the depth of the sums considered and is given by the 2nd WiTT formula. It can be also determined counting the LYNDON words of the respective index set. There are two further classes of relations: structural relations between NIELSEN-type integrals and relations due to the specific structure of FEYNMAN diagrams which lead to a considerable reduction of the set of basic functions. The relations derived can be used to simplify results of higher order calculations in QED and QCD. We also report on results calculating the 16th non-singlet moment of unpolarized structure functions at 3-loop order in the $\overline{\mathrm{MS}}$ scheme.

\section{INTRODUCTION}

Multiple nested alternating and non-alternating harmonic sums $S_{a_{1}, \ldots, a_{n}}(N)[1-3]$ emerge in perturbative higher order calculations within QED and QCD for massless fermions,

$$
\begin{aligned}
S_{a_{1}, \ldots, a_{n}}(N)= & \sum_{k_{1}=1}^{N} \sum_{k_{2}=1}^{k_{1}} \ldots \sum_{k_{n}=1}^{k_{n-1}} \frac{\operatorname{sign}\left(a_{1}\right)^{k_{1}}}{k_{1}^{\left|a_{1}\right|}} \\
& \ldots \frac{\operatorname{sign}\left(a_{n}\right)^{k_{n}}}{k_{n}^{\left|a_{n}\right|}}
\end{aligned}
$$

Here, $a_{k}$ are positive or negative integers and $N$ is a positive even or odd integer depending on the observable under consideration. One calls $n$ the depth and $\sum_{k=1}^{n}\left|a_{k}\right|$ the weight of a harmonic sum. Harmonic sums are associated to MELLin transforms of real functions or SCHWARTZ-distributions $f(x) \in \mathcal{S}^{\prime}[0,1][4]$

$$
S_{a_{1}, \ldots, a_{n}}(N)=\int_{0}^{1} d x x^{N} f_{a_{1}, \ldots, a_{n}}(x)
$$

which emerge in field theoretic calculations. Finite harmonic sums are related to harmonic polylogarithms $H_{b_{1}, \ldots, b_{n}}(x)$ [5]. Their $1 /(1 \pm$ $x$ )-weighted MELLIN transform yields harmonic sums. The inverse MeLLin transform relates the harmonic sums to functions of NIELSEN integrals [6] of the variable $x$ at least for all sums of weight $w \leq 4$ as shown in [3], and associated generalizations for higher weight. NIELSEN integrals are a generalization of the usual polylogarithms [7]. In the limit $N \rightarrow \infty$ the convergent multiple harmonic sums, i.e. those where $a_{1} \neq 1$, yield (multiple) Zeta-values $\zeta_{a_{1}, \ldots, a_{n}}$, which are also called EULER-ZAGIER sums [8]. A generalization of both harmonic polylogarithms and the EULER-ZAGIER sums are the nested $Z_{-}$ sums [9], which form a HopF algebra [10,11] and are related to GONCHAROV's multiple polylogarithms [12]. For a recent review see [13].

Higher order calculations in massless field theories are either performed in MELLIN- $N$ space referring to harmonic sums or in the space of the momentum fractions $x$ representing the results in terms of NiELSEN-type integrals. The principal complexity is determined by the amount of possible terms contributing. In the case of the 2loop coefficient functions in momentum fraction space [14] 77 different functions occurred, cf. [3]. 
This number compares to the amount of all possible different nested harmonic sums up to weight $w=4,80=3^{w}-1$. For the 3-loop anomalous dimensions [15] one expects the contribution of a wide class of the $w=5$ harmonic sums and for the 3-loop coefficient functions of the $w=6$ harmonic sums, which means 242 or 728 sums, respectively. These sums are not independent but are connected by different kind of relations. In the present paper we summarize a first class of relations recently being discussed in Ref. [16], the socalled algebraic relations. It turns out that these relations emerge from the index-structure and the multiplication relation of the objects considered and are widely independent of other properties of the harmonic sums. In this way an equivalence class of even more objects is defined having the same properties or can be found as special cases thereof. One example is the set of the harmonic polylogarithms [5].

To obtain manageable expressions it is of importance to apply all these relations through which the number of basic functions to be referred to is considerably reduced. A further reduction of the number of basic functions follows due to structural relations [17] and the symmetry of FEynman amplitudes.

Experience shows that the MELLIN space representation yields simpler expressions in general [18], which would not be seen easily working in $x$ space. To obtain as simple as possible expressions is of special importance because of the fact that data-analyses require compact results in $x$-space, which can be obtained using analytic continuations for the basic sums [19] and performing a single numeric MELLIN inversion [20] for the whole problem. Since the evaluation of precise analytic continuations needs special effort any possible reduction carried out before is of help.

\section{RELATIONS BETWEEN HARMO- NIC SUMS}

There are three types of relations between harmonic sums.

1. Algebraic relations due to the shuffle algebra of harmonic sums under their multipli- cation, cf. $[16,21,22]$.

2. Relations implied by structural relations of NiELSEN-type integrals [17], which are related to harmonic sums via a MELLIN transforms after being weighted with the denominators $1 /(1 \pm x)$

3. Symmetry relations encoded in FEYNMAN diagrams for the respective quantities, which cancel a series of harmonic sums present in individual diagrams via algebraic relations.

The product of two finite harmonic sums (1) yields

$$
\begin{aligned}
& S_{a_{1}, \ldots, a_{n}}(N) \cdot S_{b_{1}, \ldots, b_{m}}(N) \\
& =\sum_{l_{1}=1}^{N} \frac{\operatorname{sign}\left(a_{1}\right)^{l_{1}}}{l_{1}^{\left|a_{1}\right|}} S_{a_{2}, \ldots, a_{n}}\left(l_{1}\right) S_{b_{1}, \ldots, b_{m}}\left(l_{1}\right) \\
& +\sum_{l_{2}=1}^{N} \frac{\operatorname{sign}\left(b_{1}\right)^{l_{2}}}{l_{2}^{\left|b_{1}\right|}} S_{a_{1}, \ldots, a_{n}}\left(l_{2}\right) S_{b_{2}, \ldots, b_{m}}\left(l_{2}\right) \\
& -\sum_{l=1}^{N} \frac{\left[\operatorname{sign}\left(a_{1}\right) \operatorname{sign}\left(b_{1}\right)\right]^{l}}{l^{\left|a_{1}\right|+\left|b_{1}\right|}} S_{a_{2}, \ldots, a_{n}}(l) S_{b_{2}, \ldots, b_{m}}(l) .
\end{aligned}
$$

We introduce the shuffle product $\amalg$ of a single and a general finite harmonic sum

$$
\begin{aligned}
& S_{a_{1}}(N) \sqcup S_{b_{1}, \ldots, b_{m}}(N)=S_{a_{1}, b_{1}, \ldots, b_{m}}(N) \\
& +S_{b_{1}, a_{1}, b_{2}, \ldots, b_{m}}(N)+\ldots+S_{b_{1}, b_{2}, \ldots, b_{m}, a_{1}}(N)
\end{aligned}
$$

which is a linear combination of the sums of depth $m+1$. The shuffle product of two harmonic sums of depth $n$ and $m, S_{a_{1}, \ldots, a_{n}}(N)$ and $S_{b_{1}, \ldots, b_{m}}(N)$, is the sum of all harmonic sums of depth $m+n$ in the index set of which $a_{i}$ occurs left of $a_{j}$ for $i<j$ and likewise for $b_{k}$ and $b_{l}$ for $k<l$. As an example the shuffle product of two threefold harmonic sums is given by

$$
\begin{aligned}
& S_{a_{1}, a_{2}, a_{3}}(N) \amalg S_{a_{4}, a_{5}, a_{6}}(N)= \\
& \quad S_{a_{1}, a_{2}, a_{3}, a_{4}, a_{5}, a_{6}}(N)+S_{a_{1}, a_{2}, a_{4}, a_{3}, a_{5}, a_{6}}(N) \\
& +S_{a_{1}, a_{2}, a_{4}, a_{5}, a_{3}, a_{6}}(N)+S_{a_{1}, a_{2}, a_{4}, a_{5}, a_{6}, a_{3}}(N) \\
& +S_{a_{1}, a_{4}, a_{2}, a_{3}, a_{5}, a_{6}}(N)+S_{a_{1}, a_{4}, a_{2}, a_{5}, a_{3}, a_{6}}(N) \\
& +S_{a_{1}, a_{4}, a_{2}, a_{5}, a_{6}, a_{3}}(N)+S_{a_{1}, a_{4}, a_{5}, a_{6}, a_{2}, a_{3}}(N) \\
& +S_{a_{1}, a_{4}, a_{5}, a_{2}, a_{6}, a_{3}}(N)+S_{a_{1}, a_{4}, a_{5}, a_{2}, a_{3}, a_{6}}(N) \\
& +S_{a_{4}, a_{5}, a_{6}, a_{1}, a_{2}, a_{3}}(N)+S_{a_{4}, a_{5}, a_{1}, a_{6}, a_{2}, a_{3}}(N) \\
& +S_{a_{4}, a_{5}, a_{1}, a_{2}, a_{6}, a_{3}}(N)+S_{a_{4}, a_{5}, a_{1}, a_{2}, a_{3}, a_{6}}(N)
\end{aligned}
$$




$$
\begin{aligned}
& +S_{a_{4}, a_{1}, a_{5}, a_{6}, a_{2}, a_{3}}(N)+S_{a_{4}, a_{1}, a_{5}, a_{2}, a_{6}, a_{3}}(N) \\
& +S_{a_{4}, a_{1}, a_{5}, a_{2}, a_{3}, a_{6}}(N)+S_{a_{4}, a_{1}, a_{2}, a_{3}, a_{5}, a_{6}}(N) \\
& +S_{a_{4}, a_{1}, a_{2}, a_{5}, a_{3}, a_{6}}(N)+S_{a_{4}, a_{1}, a_{2}, a_{5}, a_{6}, a_{3}}(N)
\end{aligned}
$$

Finally one establishes a system of linear equations in which the linear elements of the shuffle products form the variables and a polynomial out of harmonic sums of lower depth forms the inhomogeneity. We furthermore consider all index permutations. This system contains all algebraic relations. In Ref. [16] all solutions for harmonic sums up to depth $d=6$ were given. This complies to the level of sophistication needed to reduce the corresponding relations which emerge for massless 3-loop coefficient functions.

\section{NUMBER OF ALGEBRAICALLY IN- DEPENDENT HARMONIC SUMS}

Let us consider the index set of a harmonic sum of depth $d$. One may consider this set as a word $w$ or a non-commutative product of letters of an ordered alphabet $\mathfrak{A}=\{a, b, c, d, \ldots\}$. Any word can be decomposed into three parts

$$
w=p x s
$$

a prefix $p$, a suffix $s$, and the remainder part $x$. Among all words $w$ the LYNDON words, cf. e.g. [23], are those being smaller than any of its suffixes.

According to a Theorem by RADFORD [24] the shuffle algebra discussed above is freely generated by the LYNDON words, i.e. the length of its basis is given by the number of LYNDON words. We would like to count the number of LYNDON words for index sets, where the same letters can emerge repeatedly. The corresponding relation is due to WitT [25] and will be called 2nd WitT formula

$$
l_{n}\left(n_{1}, \ldots, n_{q}\right)=\frac{1}{n} \sum_{d \mid n_{i}} \mu(d) \frac{\left(\frac{n}{d}\right) !}{\left(\frac{n_{1}}{d}\right) ! \ldots\left(\frac{n_{q}}{d}\right) !},
$$

with $n=\sum_{k=1}^{q} n_{k}$. Here $\mu(d)$ denotes the MöBIUs function. One may derive $l_{n}\left(n_{1}, \ldots, l_{q}\right)$ using the generating functional

$\frac{1}{1-x_{1}-\ldots-x_{n_{q}}}=\prod_{n=1}^{\infty}\left(\frac{1}{1-\sum_{k=1}^{q} x_{k}^{d_{k}}}\right)^{l_{n}\left(n_{i}\right)}$

Note that (7) is related to the GAUss-WiTT relation mentioned by HoFFMAN [21] for the number of basic multiple Zeta-values of weight $w$ for $\forall n_{i}>0$ if all cases for fixed weight are summed over. An even more strict relation in the inclusive case has been conjectured by ZAGIER [8] and Broadhurst and Kreimer [26] in the case of multiple Zeta-values and verified up to $w=12$.

Let us come back to Eq. (7). We can draw some immediate conclusions out of this relation. If the numbers $n_{i}$ have no common divisor larger than 1 , the number of the basis elements compared to the number of all objects equals $1 / d$, where $d$ denotes the depth of the index set. In case of common divisors larger than 1 we checked that the basis is always shorter for all depths up to $d=10$, see $[16]$.

\begin{tabular}{||r||r|r|l||}
\hline \hline Weight & \# Sums & \# Basic Sums & Fraction \\
\hline \hline 1 & 2 & 0 & 0.0 \\
2 & 8 & 1 & 0.1250 \\
3 & 26 & 7 & 0.2692 \\
4 & 80 & 23 & 0.2875 \\
5 & 242 & 69 & 0.2851 \\
6 & 728 & 183 & 0.2513 \\
\hline \hline
\end{tabular}

\section{OTHER RELATIONS}

Further to the algebraic relations structural relations between the generating functions of harmonic sums, the $1 /(1 \pm x)$ weighted Nielsen-type integrals, exist. These relations reduce the number of basic harmonic sums further [17]. As an example we mention the relation

$$
\begin{aligned}
\frac{1}{2} \frac{\mathrm{Li}_{2}^{2}\left(x^{2}\right)}{1-x^{2}} & =\frac{\mathrm{Li}_{2}(x)}{1-x}+\frac{\mathrm{Li}_{2}(-x)}{1-x} \\
& +\frac{\mathrm{Li}_{2}(x)}{1+x}+\frac{\mathrm{Li}_{2}(-x)}{1+x}
\end{aligned}
$$

The structure of Feynman diagrams is furthermore selective on the type of harmonic sums which may occur for the final physical results. As mentioned before, $x$-space calculations of the 
two-loop WiLson coefficients resulted in the occurrence of nearly as many different functions as combinatorially possible. Studying the MeLLin transforms of these functions in detail $[18,27]$ one finds that a series of harmonic sums occurs with a partial index symmetry such that certain classes of basic functions do not contribute. In the case of two loop WILSON coefficients the function

$$
\frac{\ln (1+x)}{1+x}
$$

which is mathematically irreducible, does not contribute, although it is of weight 2 and functions up to weight 4 span the basis. Likewise other irreducible lower-weight functions, which cannot be eliminated by structural relations do not occur. The function (9) is, however, instrumental to build the basis spanning the set of functions required for the 3-loop anomalous dimensions.

\section{A QUADRATIC LAW ?}

The final analysis of the anomalous dimensions and WILSON coefficients $[18,27,28]$ shows that one may come to the following representation :

1. Single harmonic sums $S_{ \pm k}(N)$ and their analytic continuation $\propto \psi^{k-1}(z)$ are regarded as well-known functions. Likewise any derivative of the analytic continuation of the MeLLin transforms over $[0,1]$ of the basic functions introduced subsequently are considered to be trivial.

2. The analytic continuation of the MeLLiN transforms of the basic functions are meromorphic functions with poles at the integers bound from above by a positive number. They can be represented by factorial series [29].

3 . The one-loop anomalous dimensions require $\mathrm{w}=1$ harmonic sums, likewise the $1-$ loop WILSON coefficient are expressed by at most $w=2$ harmonic sums, which are all trivial in the above sense.

4. The two-loop anomalous dimension are represented as polynomials of trivial functions but the MELLin transform of one nontrivial function of up to $w=3$

$$
\frac{\operatorname{Li}_{2}(x)}{1+x}, \quad[1]
$$

5. The two-loop WiLSON coefficients are represented as polynomials of the MELLIN transform of the functions mentioned and the four additional basic functions

$$
\frac{\mathrm{Li}_{2}(x)}{1-x}, \frac{\mathrm{Li}_{3}(x)}{1+x}, \frac{\mathrm{S}_{1,2}(x)}{1 \pm x}
$$

6. The three-loop anomalous dimensions are represented as polynomials of the MELLIN transform of the functions mentioned and the nine additional basic functions

$$
\begin{aligned}
& \frac{\ln (1+x)}{1+x}, \frac{\operatorname{Li}_{4}(x)}{1 \pm x}, \\
& \frac{\mathrm{S}_{1,3}(x)}{1+x}, \frac{\mathrm{S}_{2,2}(x)}{1 \pm x}, \\
& \frac{\mathrm{S}_{2,2}(-x)-\mathrm{Li}_{2}^{2}(-x) / 2}{1 \pm x}, \frac{\mathrm{Li}_{2}^{2}(x)}{1+x}
\end{aligned}
$$

In this way the number of basic functions contributing to the single scale quantities discussed up to weight $w=5$ leads to the following reduction from the combinatorial complexity

$$
2 \cdot 3^{w-1} \rightarrow \theta(w-2)[w-2]^{2},
$$

cf. [28], i.e. the number of basic elements grows only quadratically, rather than exponentially. This relation will be examined for the case of three-loop coefficient functions, i.e. for $w=6$, very soon.

\section{THE 16th MOMENT OF THE NON- SINGLET THREE LOOP ANOMA- LOUS DIMENSION OF $F_{1}\left(x, Q^{2}\right)$}

Previous fixed-moment calculations of anomalous dimensions and WILSON coefficients extended to the moments $N=14$ for the nonsinglet structure function $F_{1, L}\left(x, Q^{2}\right)$ and $N=$ 13 for $x F_{3}\left(x, Q^{2}\right)$ [15]. Weeks before the calculation of the complete result for the 3-loop 
non-singlet anomalous dimensions [31] was finished ${ }^{1}$ the calculation of the 16 th moment of the non-singlet anomalous dimension of the structure function $F_{1}\left(x, Q^{2}\right)$ and the WILSON coefficients for $F_{2, L}\left(x, Q^{2}\right)$ was started using the MINCER algorithm [30]. The result of this calculation provides an independent test of the complete results. The calculation was performed using several powerPC's partially linked to a 4.2 Tbyte raid system. ${ }^{2}$ The calculational time for both the projections $g_{\mu \nu}$ and $P_{\mu} P_{\nu}$ of the forward Compton amplitude amounted to $564 \mathrm{CPU}$ days. The 16 th moment of the non-singlet anomalous dimension for $F_{1}^{\mathrm{NS}}\left(x, Q^{2}\right)$ reads, cf. [32]:

$$
\begin{aligned}
\gamma_{16}^{(0)}= & \frac{64419601}{6126120} C_{F} \\
\gamma_{16}^{(1)}= & -\frac{1176525373840303}{112588038763200} C_{F} N_{F} \\
& +\frac{21546159166129889}{484994628518400} C_{F} C_{A} \\
& -\frac{3689024452928781382877}{459818557352009856000} C_{F}{ }^{2}(15
\end{aligned}
$$

$$
\begin{aligned}
\gamma_{16}^{(2)}= & \left(\frac{59290512768143}{1563722760600} \zeta_{3}\right. \\
- & \left.\frac{58552930270652300886778705063429867}{3451337970612452534317096673280000}\right) \\
& \cdot C_{F}^{3} \\
+ & \left(-\frac{15018421824060388659436559}{579371382263532418560000}\right. \\
- & \left.\frac{64419601}{765765} \zeta_{3}\right) C_{F} C_{A} N_{F} \\
+ & \left(\frac{1670423728083984207878825467}{6488959481351563087872000}\right. \\
+ & \left.\frac{59290512768143}{3127445521200} \zeta_{3}\right) C_{F} C_{A}{ }^{2} \\
- & \frac{5559466349834573157251}{2069183508084044352000} C_{F} N_{F}{ }^{2}
\end{aligned}
$$

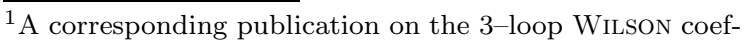
ficients will appear later.

${ }^{2}$ We thank S. Wiesand and P. Wegner for technical assistance and U. Gensch and C. Spiering for their support of this calculation.
}

$$
\begin{aligned}
& +\left(-\frac{1229794646000775781127856064477}{30335885575318557435801600000}\right. \\
& \left.-\frac{59290512768143}{1042481840400} \zeta_{3}\right) C_{F}{ }^{2} C_{A} \\
& -\quad+\left(\frac{71543599677985155342551355451}{938967886855098206346240000}\right. \\
& \left.+\frac{64419601}{765765} \zeta_{3}\right) C_{F}{ }^{2} N_{F}
\end{aligned}
$$

and agrees with the result in [31] for $N=16$. The corresponding moments of the WILSON coefficients $C_{2, L}\left(x, Q^{2}\right)$ are given in [32].

\section{CONCLUSIONS}

The product of finite alternating or nonalternating harmonic sums is given by the shuffle product of harmonic sums and polynomials of harmonic sums of lower depth. These representations imply algebraic relations between the harmonic sums. If one considers all harmonic sums associated to an index set $\left\{a_{1}, \ldots, a_{k}\right\}$ one may express these sums by a number of basic sums. It turns out that this number is given by the $2 \mathrm{nd}$ WITT formula which counts the number of LYNDON words corresponding to the respective index set. The set of these LYNDON words generates in this sense all harmonic sums of this class freely. By solving the corresponding linear equations we derived the explicit representation of all harmonic sums up to depth $d=6$ without specifying the indices numerically and gave all expression which are structurally needed to express the sums up to weight $w=6$. The counting relations for the basis of the finite harmonic sums were given up to depth $d=10$. The relations derived hold likewise for other mathematical objects obeying the same multiplication relation or a simpler one, which is being contained, as that for harmonic polylogarithms. This is due to the fact that the relations derived depend on the index set and the multiplication relation but on no further properties of the objects considered.

The ratio of the number of basic sums for a given index set and the number of all sums is mainly determined by the depth $d$ rather than 
the weight of the respective sums, due to the prefactor $1 / d$ in the WitT formula. Modifications occur due to common non-trivial divisors of the numbers of individual indices in the set being considered. Up to $d=10$ we showed that the fraction of basic sums is always $\leq 1 / d$ compared to all sums. The use of these algebraic relations leads to a considerable reduction in the set of functions needed to express the results of higher order calculations in massless QED and QCD and related subjects. Further reductions are due to structural relations and symmetries in the set of Feynman amplitudes calculated. For practical applications such as the description of the QCD scaling violation of the structure functions in deeply inelastic scattering the harmonic sums occurring in the MELLin $N$ space calculation have to be translated to $x$-space by the inverse MeLLin transform. For this reason the respective harmonic sums have to be analytically continued in the argument $N$ to complex values, which requires a high effort using numerical procedures. It is therefore recommended to use as many as possible relations between the $N$ space objects before the last step is being performed.

The 16th moment for the parity-conserving non-singlet structure function was calculated. It provides a test on the complete result, which has been derived recently for the anomalous dimensions, and on the upcoming result for the coefficient functions.

Acknowledgment. This paper was supported in part by DFG Sonderforschungsbereich Transregio 9, Computergestützte Theoretische Physik and EU grant HPRN-CT-2000-00149. I would like to thank S. Moch, V. Ravindran, T. Riemann and J. Vermaseren for discussions.

\section{REFERENCES}

1. A. Gonzalez-Arroyo, C. Lopez, and F.J. Yndurain, Nucl. Phys. B153 (1979) 161;

A. Gonzalez-Arroyo and C. Lopez, Nucl. Phys. B166 (1980) 429.

2. J.A.M. Vermaseren, Int. J. Mod. Phys. A14 (1999) 2037.

3. J. Blümlein and S. Kurth, DESY 97-160, hep-ph/9708388; Phys. Rev. D60 (1999) 014018.

4. L. Schwartz, Théorie des Distributions (Hermann and Cie, Paris, 1951), Vols. I and II; V.S. Vladimirov, Gleichungen der Mathematischen Physik, (DVW, Berlin, 1972);

K. Yoshida, Functional Analysis (Springer, Berlin, 1978).

5. E. Remiddi and J. Vermaseren, Int. J. Mod. Phys. A15 (2000) 725.

6. N. Nielsen, Nova Acta Leopold. XC, 121 (1909);

S. Kölbig, Siam J. Math. Anal. 17 (1986) 1232.

7. L. Lewin, Dilogarithms and Associated Functions (Mac-Donald, London, 1958); Polylogarithms and Associated Functions, (North Holland, New York, 1981);

A. Devoto and D.W. Duke, Riv. Nuovo Cim. 7 N6 (1984) 1.

8. L. Euler, Novi Comm. Acad. Sci. Petropol. 20 (1775) 140;

D. Zagier, First European Congress of Mathematics, (Birkhäuser, Boston, 1994), Vol.II, p. 497.

9. S. Moch, P. Uwer, and S. Weinzierl, J. Math. Phys. 43 (2002) 3363;

S. Weinzierl, Comput. Phys. Commun. 145 (2002) 357.

10. H. Hopf, Ann. of Math. 42 (1941) 22;

J. Milner and J. Moore, Ann. of Math. 81 (1965) 211;

M.E. Sweedler, Hopf Algebras, (Benjamin, New York, 1969).

11. C. Kassel, Quantum Groups, (Springer, Berlin, 1995).

12. A.B. Goncharov, Math. Res. Lett. 5 (1998) 497, http://www.math.uiuc.edu/K-Theory/0297.

13. M. Waldschmidt, J. Théor. Nombres Bordeaux 12 (2000) 581; Multiple polylogarithms, Lectures at the Institute of Math. Sciences (Chennai, Nov. 2000); updated version http://www.math.jussieu.fr/ 〜miw/ articles/ps/mpl.ps; V.V. Zudilin, Uspekhi Mat. Nauk (Russian Math. Surveys) $\mathbf{5 8}$ (2003) 3.

14. W.L van Neerven and E.B. Zijlstra, Nucl. 
Phys. B382 (1992) 11.

15. S.A. Larin, T. van Ritbergen, and J.A.M. Vermaseren, Nucl. Phys. B427 (1994) 41;

S.A. Larin, P. Nogueira, T. van Ritbergen, and J.A.M. Vermaseren Nucl. Phys. B492 (1997) 338;

A. Retey and J.A.M. Vermaseren, Nucl. Phys. B604 (2001) 281.

16. J. Blümlein, Comput. Phys. Commun. 159 (2004) 19.

17. J. Blümlein, DESY 04-064.

18. J. Blümlein and S. Moch, in preparation.

19. J. Blümlein, Comput. Phys. Commun. 133 (2000) 76.

20. J. Blümlein and A. Vogt, Phys. Rev. D57 (1998) 1; D58 (1998) 014020.

21. M.E. Hoffman, J. Algebra, 194 (1997) 477.

22. M.E. Hoffman, math.QA/0406589 and these Proceedings; Pacific J. Math. 152 (1992) 275; J. Algebraic Combin. 11 (2000) 49; math.QA/9907173; math.NT/0401319;

Ngoc Minh Hoang, these Proceedings and references therein.

23. C. Reutenauer, Free Lie Algebras, (Calendron Press, Oxford, 1993).

24. D.E. Radford, J. Algebra, 58 (1979) 432.

25. E. Witt, Journ. Reine und Angew. Mathematik, 177 (1937) 152; Math. Zeitschr. 64 (1956) 195.

26. D.J. Broadhurst and D. Kreimer, Phys. Lett. B393 (1997) 403.

27. J. Blümlein and V. Ravindran, these Proceedings and in prepartion.

28. J. Blümlein and S. Moch in preparation.

29. N. Nielsen, Handbuch der Theorie der Gammafunktion, (Teubner, Leipzig, 1906);

E. Landau, Über die Grundlagen der Theorie der Fakultätenreihen, S.-Ber. math.-maturw. Kl. Bayerische Akad. Wiss. München, 36 (1906) 151.

30. S. A. Larin, F. V. Tkachev, and J. A. M. Vermaseren, NIKHEF-H-91-18.

31. S. Moch, J. Vermaseren and A. Vogt, Nucl. Phys. B688 (2004) 101.

32. J. Blümlein and J. Vermaseren, in preparation. 